\title{
Prognostic significance of diastolic blood pressure in patients with heart failure with preserved ejection fraction
}

\author{
Aya Fuchida ${ }^{1}$-Sho Suzuki ${ }^{1}$ (1) $\cdot$ Hirohiko Motoki ${ }^{2} \cdot$ Yusuke Kanzaki $^{1} \cdot$ Takuya Maruyama $^{1} \cdot$ Naoto Hashizume $^{1}$. \\ Ayako Kozuka $^{1} \cdot$ Kumiko Yahikozawa $^{1} \cdot$ Koichiro Kuwahara ${ }^{2}$
}

Received: 18 September 2020 / Accepted: 15 January 2021 / Published online: 2 February 2021

(c) The Author(s) 2021

\begin{abstract}
Although systolic blood pressure (SBP) is routinely considered when treating acute heart failure (HF), diastolic blood pressure (DBP) is hardly been assessed in the situation. There are no previous studies regarding the predictive value of DBP in elderly patients with HF with preserved ejection fraction (HFpEF) in Japan. This study aimed to investigate the prognostic significance of DBP in patients with acute decompensated HFpEF. We analyzed data of all HFpEF patients admitted to Shinonoi General Hospital for HF treatment between July 2016 and December 2018. We excluded patients with acute coronary syndrome and severe valvular disease. Patients were divided into two groups according to their median DBP; the low DBP group (DBP $\leq 77 \mathrm{mmHg}, n=106)$ and the high DBP group (DBP $>77 \mathrm{mmHg}, n=100$ ). The primary outcome was HF readmission. In 206 enrolled patients (median 86 years), during a median follow-up of 302 days, the primary outcome occurred in 48 patients. The incidence of HF readmission was significantly higher in the low DBP group (33.0\% vs $18.5 \%$, $p=0.024)$. In Kaplan-Meier analysis, low DBP predicted HF readmission (Log-rank test, $p=0.013$ ). In Cox proportional hazard analysis, low DBP was an independent predictor of HF readmission after adjustment for age, sex, SBP, hemoglobin, serum albumin, serum creatinine, B-type natriuretic peptide, renin-angiotensin system inhibitors, calcium channel blockers, left ventricular ejection fraction, coronary artery disease, and whether they live alone (hazard ratio, 2.229; 95\% confidence interval, $1.021-4.867 ; p=0.044)$. Low DBP predicted HF readmission in patients with HFpEF.
\end{abstract}

Keywords HFpEF · DBP · Heart failure readmission · Diastolic blood pressure

\section{Introduction}

Improvements in cardiovascular survival rates and progressive aging of the population have led to an increase in elderly patients with heart failure with preserved ejection fraction (HFpEF) [1-4]. The number of elderly HFpEF patients will continue to increase annually in Japan. Given the high cost of inpatient heart failure (HF) treatment, HF places a major burden on the public health system and has an associated economic impact. Furthermore, repeating HF readmission could worsen the activity of daily living (ADL) and quality

Sho Suzuki

jersey0042@gmail.com

1 Department of Cardiovascular Medicine, Minaminagano Medical Center, Shinonoi General Hospital, Ai 666-1 Shinonoi, Nagano 388-8004, Japan

2 Department of Cardiovascular Medicine, Shinshu University School of Medicine, Matsumoto, Japan of life (QOL) in elderly HF patients. Considering these facts, preventing HF readmission in elderly HF patients would be helpful for both patients and the economic cost.

The fundamental pathophysiological mechanism of HFpEF remains undefined, and the tools for risk-stratification are needed to improve the management of these patients. In clinical practice, we encounter elderly patients with low diastolic blood pressure (DBP) in HFpEF. Decreased DBP is demonstrated to have an association with arterial stiffening, as implied by Sleight's hypothesis many years ago [5]. The relation between DBP and cardiovascular events could be attributed to the decline in DBP as a consequence of stiffing of the large arteries in elderly patients with HFpEF. Although systolic blood pressure (SBP) is routinely considered when treating acute heart failure [6], DBP is hardly been assessed in the situation. Several recent studies have reported the association between low DBP and adverse outcomes in stable HFpEF without adjusting for SBP [7, 8]. However, there are no previous studies regarding the 
predictive value of low DBP on HF readmission in an elderly Japanese HFpEF cohort. Against this background, we aimed to identify the prognostic significance of low DBP in elderly patients with acute decompensated HFpEF in a retrospective cohort study.

\section{Materials and methods}

\section{Study design}

This was a retrospective, single-center cohort study. The cohort included patients admitted to Shinonoi General Hospital between July 2016 and December 2018 with a primary diagnosis of acute decompensated HFpEF. Patients with acute coronary syndrome, HF with reduced ejection fraction, and severe valvular heart disease were excluded (Fig. 1). The study and all its protocols were approved by the Shinonoi General Hospital Ethics Committee, and informed consent was obtained. We collected data on clinical characteristics, medical history, major risk factors for HF, comorbidities, laboratory tests, electrocardiography, echocardiography, available angiographic data, medications, treatment and clinical events during hospitalization, and post-discharge follow-up findings.

Blood pressure was defined as the average of two data in sinus rhythm, five data in atrial fibrillation measured in oscillometric method collected at admission, either in the upright or supine position. Acute decompensated HF was defined by the Framingham criteria [9]. The diagnosis of HF and acute coronary syndrome was made by the treating clinicians using all available symptoms, laboratory, electrocardiogram, echocardiography, and coronary angiogram data. Transthoracic echocardiography was performed using standardized equipment (HD15 Ultrasound Machine, Royal Philips, Amsterdam, Noord-Holland, the Netherlands; CX50 xMatrix, Royal Philips, Amsterdam, Noord-Holland, the Netherlands; Toshiba Artida, CANON Medical Systems Corporation, Otawara, Tochigi, Japan) in compliance with the recommendations of the American Society of Echocardiography and the European Association of Cardiovascular Imaging $[10,11]$. The biplane modified Simpson's method was used to measure left ventricular ejection fraction (LVEF). Echocardiography was produced within $24 \mathrm{~h}$ after admission, and HFpEF was defined as HF with LVEF $\geq 50 \%$.

All data were fully anonymized before access. The investigation is consistent with the principles outlined in the Declaration of Helsinki.

\section{Follow-up}

The primary outcome was hospitalization during follow-up due to worsening HF. Incidents were validated by chart view.

\section{Statistical analysis}

Continuous variables are summarized as the mean \pm standard deviation if normally distributed and as the median and interquartile range if non-normally distributed. Normality was assessed using the Shapiro-Wilk $W$-test. Comparisons of baseline characteristics were made with a contingency table and the Pearson $\chi^{2}$ test for categorical variables, the $t$ test for normally distributed continuous variables, and Mann Whitney $U$ test for non-normally distributed continuous variables. Spearman's rank correlation method was used as a nonparametric measure of the association

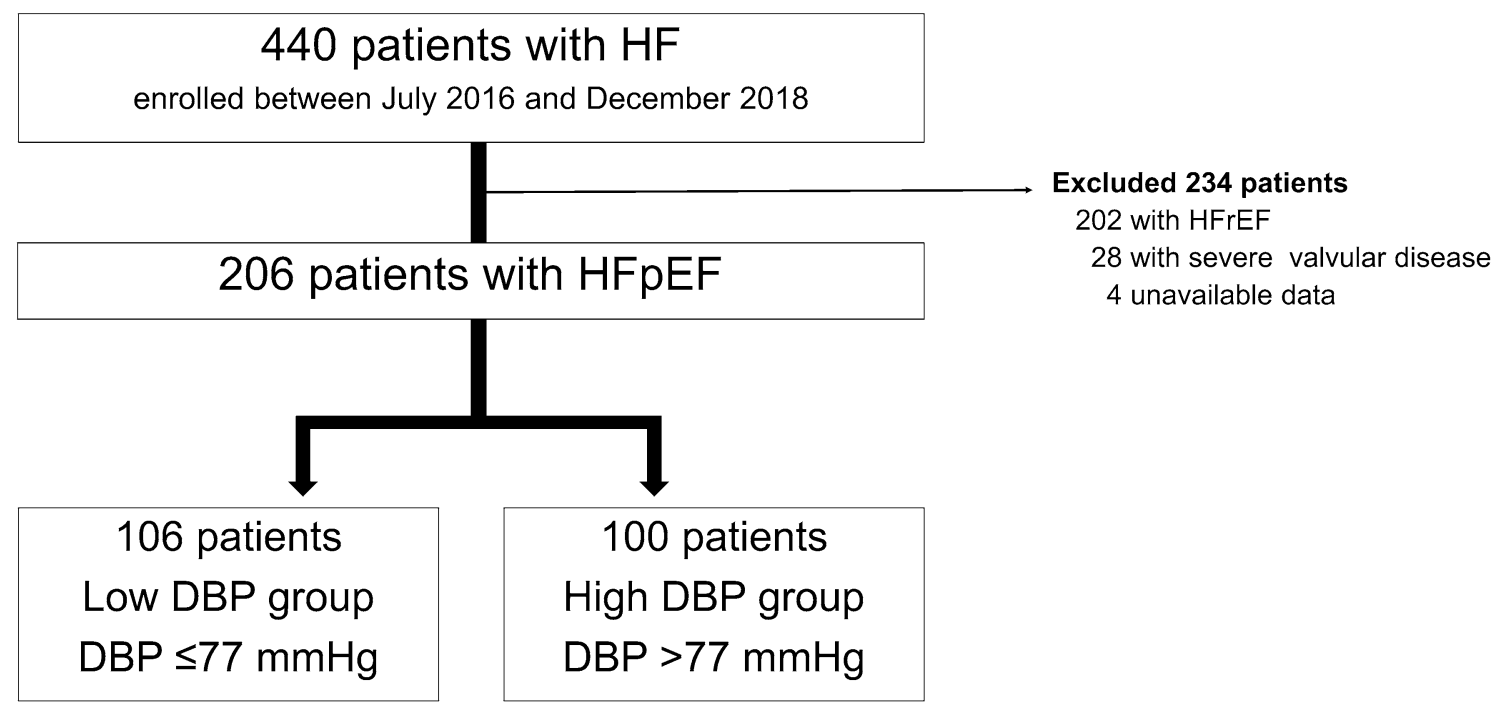

Fig. 1 Patient flow chart 
between DBP and clinical indices. Patients were then divided into two groups according to the median DBP level: the high DBP group (DBP $>77 \mathrm{mmHg}, n=100$ ), and the low DBP group (DBP $\leq 77 \mathrm{mmHg}, n=106$ ). Kaplan-Meier survival plots were calculated from baseline to the time of HF readmission and compared using the log-rank test. Cox proportional-hazards analysis was used to evaluate the independent prognostic utility of DBP. The covariates used were age, sex, SBP, hemoglobin, serum albumin, serum creatinine, B-type natriuretic peptide (BNP), renin-angiotensin system inhibitors (RASi), calcium channel blockers (CCB), LVEF, coronary artery disease (CAD), and whether they live alone. A $p$-value of $<0.05$ was considered statistically significant. All statistical analyses were performed using SPSS Statistics software for Windows Version 26 (IBM Corp., Armonk, NY, USA).

\section{Results}

\section{Study population}

We enrolled 206 patients (median age 86 years; female, $58 \%$ ). Twenty patients died during hospitalization. The baseline characteristics are shown in Table 1. Patients with low DBP had lower SBP, heart rate, body mass index, $\mathrm{BNP}$, hemoglobin, and higher serum creatinine than those with high DBP. Anticoagulants were more frequently prescribed in the low DBP group. After analysis, there were no strong correlations between DBP and clinical indices (Table 2).

\section{The prognostic significance of diastolic blood pressure}

During a median follow-up of 302 days [interquartile range 119-636], 48/186 (25.8\%) patients experienced HF readmission. The low DBP group was related to an increased risk of HF readmission [low DBP group 33.0\% (31/94) vs high DBP group: 18.5\% (17/92), $p=0.024$ ] (Fig. 2). In the Kaplan-Meier analysis, the low DBP group predicted HF readmission (Log rank test, $p=0.013$ ) (Fig. 3). In the multivariate Cox proportional hazards analysis, the low DBP group was an independent predictor of HF readmission after adjustment for age, sex, SBP, hemoglobin, serum albumin, serum creatinine, BNP, RASi, CCB, LVEF, CAD, and whether they live alone (hazard ratio $2.229 ; 95 \%$ confidence interval 1.021-4.867; $p=0.044$ ) (Table 3). The result was consistent upon the exclusion of CAD (hazard ratio 2.229; 95\% confidence interval 1.021-4.866; $p=0.044)$.

\section{Discussion}

The novel finding of the present study is that the low DBP group was significantly associated with an increased risk of HF readmission in extremely elderly patients with acute decompensated HFpEF. This association was independent of other well-established HF risk factors, including age, BNP, renal function, serum albumin, LVEF, and importantly, SBP.

Previous studies have reported the association between low SBP and adverse outcomes in patients with HF [12, 13]. In terms of DBP, several recent studies have investigated the significant association between low DBP and poor prognosis in stable HFpEF [7, 8]. However, these studies were evaluated without adjusting for SBP, and the independent prognostic value of DBP remained unclear.

In our study, we identified that the low DBP group had a significantly higher risk of HF readmission compared with the high DBP group in elderly HFpEF patients hospitalized for acute decompensated HF. To the best of our knowledge, no other study has investigated the prognostic impact of low DBP independent of SBP in these patients.

The underlying pathophysiology of HFpEF remains unclear. A previous study reported that atrial stiffness, a result of the substantial progression of atherosclerosis, could be one of the complex mechanisms of this disease [14-16]. On the other hand, decreased DBP has been demonstrated to indicate arterial stiffening, associated with atherosclerotic progression [5, 17-19]. There is a possibility that large artery stiffening, a result of the substantial progression of atherosclerosis, could be the underlying pathophysiological mechanism of poor prognosis in elderly HFpEF patients with decreased DBP. In addition, low DBP could lead to decreased coronary perfusion pressure, which may result in myocardial damage and worsening ventricular dysfunction $[8,20,21]$. This may also be a reason for the poor prognosis in HFpEF patients with low DBP. However, these hypotheses are only speculative, and further studies are needed.

From our findings, we suggest that DBP could be a useful risk-stratification tool in cases of HFpEF. Although SBP is routinely considered when treating acute heart failure, as cardiac shock or low output syndrome defined by low SBP, DBP is hardly been assessed in the situation. We hypothesize that patients with decreased DBP could have a poor prognosis even if their SBP is preserved. Patients with low DBP at admission should receive aggressive therapy and close outpatient follow-up after discharge. However, although decreased DBP may result in an increased risk of HF readmission, this does not necessarily mean that extremely high DBP are preferred in these patients, since several previous studies have reported that high DBP was, likewise to low DBP, associated with adverse outcomes in patients with cardiovascular disease [8, 22, 23]. 
Table 1 Baseline characteristics

\begin{tabular}{|c|c|c|c|c|}
\hline Variables & $\begin{array}{l}\text { Overall population } \\
(n=206)\end{array}$ & $\begin{array}{l}\mathrm{DBP} \leq 77 \mathrm{mmHg} \\
(n=106)\end{array}$ & $\begin{array}{l}\mathrm{DBP}>77 \mathrm{mmHg} \\
(n=100)\end{array}$ & $p$-value \\
\hline Age (years) [range] & 86 [81-91] & $86[81-91]$ & $87[81-92]$ & 0.976 \\
\hline Male, sex, $n(\%)$ & $87(42)$ & $48(45)$ & $39(39)$ & 0.362 \\
\hline BMI & $22.5[19.6-25.5]$ & $22.0[19.3-24.6]$ & $23.5[20.8-25.9]$ & 0.007 \\
\hline Systolic blood pressure $(\mathrm{mmHg})$ & $137[115-154]$ & $120[102-142]$ & $146[134-166]$ & $<0.001$ \\
\hline Pulse pressure (mmHg) & 55 [42-69] & $58[43-71]$ & $54[40-63]$ & 0.243 \\
\hline Heart rate (bpm) & 85 [70-104] & 78 [65-95] & $90[72-110]$ & 0.001 \\
\hline CAD, $n(\%)$ & $43(21)$ & $22(21)$ & $21(21)$ & 0.965 \\
\hline Hypertension, $n(\%)$ & $134(65)$ & $65(61)$ & $69(69)$ & 0.248 \\
\hline Atrial fibrillation, $n(\%)$ & $130(63)$ & $73(69)$ & $57(57)$ & 0.078 \\
\hline Dyslipidemia, $n(\%)$ & $64(31)$ & $29(27)$ & $35(35)$ & 0.236 \\
\hline Diabetes mellitus, $n(\%)$ & $52(25)$ & $23(22)$ & $29(29)$ & 0.228 \\
\hline CKD, $n(\%)$ & $54(26)$ & $33(31)$ & $21(21)$ & 0.098 \\
\hline Living alone, $n(\%)$ & $28(14)$ & $14(13)$ & $14(14)$ & 0.868 \\
\hline Dementia, $n(\%)$ & $62(30)$ & $30(28)$ & $32(32)$ & 0.563 \\
\hline \multicolumn{5}{|l|}{ Echocardiographic data } \\
\hline $\operatorname{LVEF}(\%)$ & $64[60-68]$ & $64[60-68]$ & $64[60-67]$ & 0.653 \\
\hline $\mathrm{E} / \mathrm{A}$ & 1.027 [0.715-1.687] & $0.862[0.696-1.718]$ & $1.072[0.813-1.670]$ & 0.517 \\
\hline Mean E/e' & 16.26 [11.35-21.79] & $15.43[9.426-21.58]$ & 16.70 [13.24-22.67] & 0.925 \\
\hline \multicolumn{5}{|l|}{ Laboratory data } \\
\hline BNP (pg/mL) & $493[310-831]$ & $453[277-673]$ & 507 [360-934] & 0.151 \\
\hline Hemoglobin (g/dL) & $11.1[9.7-12.4]$ & $10.8[9.2-12.1]$ & $11.5[9.9-12.6]$ & 0.006 \\
\hline Serum albumin $(g / d L)$ & $3.4[3.1-3.8]$ & $3.4[3.0-3.7]$ & $3.5[3.1-3.8]$ & 0.106 \\
\hline Serum creatinine $(\mathrm{mg} / \mathrm{dL})$ & $1.01[0.73-1.44]$ & $1.11[0.78-1.62]$ & $0.91[0.68-1.27]$ & 0.018 \\
\hline $\mathrm{HbA} 1 \mathrm{c}(\%)$ & $5.9[5.6-6.4]$ & $5.9[5.5-6.4]$ & $5.9[5.6-6.4]$ & 0.594 \\
\hline $\mathrm{CRP}(\mathrm{mg} / \mathrm{dL})$ & $0.55[0.15-2.23]$ & $0.64[0.17-2.63]$ & $0.47[0.13-1.52]$ & 0.087 \\
\hline \multicolumn{5}{|l|}{ Medication } \\
\hline Antiplatelet drug, $n(\%)$ & $57(31)$ & $27(29)$ & $30(33)$ & 0.566 \\
\hline Anticoagulant, $n(\%)$ & $101(54)$ & $58(62)$ & $43(47)$ & 0.041 \\
\hline RASis, $n(\%)$ & $113(61)$ & $55(59)$ & $58(63)$ & 0.527 \\
\hline Beta-blockers, $n(\%)$ & $112(60)$ & $54(57)$ & $58(63)$ & 0.436 \\
\hline MRAs, $n(\%)$ & $98(53)$ & $45(48)$ & $53(58)$ & 0.184 \\
\hline Loop diuretic, $n(\%)$ & $156(84)$ & $77(82)$ & $79(86)$ & 0.463 \\
\hline CCBs, $n(\%)$ & $81(44)$ & $44(47)$ & $37(40)$ & 0.365 \\
\hline Statin, $n(\%)$ & $46(25)$ & $22(23)$ & $24(26)$ & 0.672 \\
\hline
\end{tabular}

Values are presented as the mean $\pm \mathrm{SD}$, median [interquartile range], or $\mathrm{n}(\%)$

$D B P$ diastolic blood pressure, $B M I$ body mass index, $C A D$ coronary artery disease, $C K D$ chronic kidney disease, $L V E F$ left ventricular ejection fraction, $E / A$ early diastolic filling velocity/atrial filling velocity ratio, E/e' early diastolic filling velocity/early diastolic velocity of the mitral annulus ratio, $B N P$ B-type natriuretic peptide, $C R P$ C-reactive protein, $R A S i$ renin-angiotensin system inhibitor, $M R A$ mineralocorticoid receptor antagonist, $C C B$ calcium channel blocker

Our study had several limitations. First, we included a small number of patients taken from a single center, and the follow-up period was short. Further research in a large cohort is necessary to verify our findings. Second, there was only a single data of DBP at the decompensated phase of HF. Serial changes or continuous measures of DBP were not evaluated 
Table 2 Univariate Spearman's rank correlation between diastolic blood pressure and clinical indices

\begin{tabular}{lrr}
\hline Variables & Spearman's Rank & $p$-value \\
\hline Age (years) & 0.029 & 0.680 \\
Male, sex & -0.064 & 0.364 \\
Systolic blood pressure (mmHg) & 0.480 & $<0.001$ \\
Hemoglobin (g/dL) & 0.183 & 0.009 \\
Serum albumin (g/dL) & 0.120 & 0.089 \\
Serum creatinine (mg/dL) & -0.165 & 0.017 \\
BNP (pg/mL) & 0.155 & 0.028 \\
RASis, $n(\%)$ & 0.046 & 0.529 \\
CCBs, $n(\%)$ & -0.066 & 0.367 \\
LVEF $(\%)$ & -0.018 & 0.793 \\
CAD, $n(\%)$ & 0.003 & 0.966 \\
Living alone, $n(\%)$ & 0.012 & 0.869 \\
\hline
\end{tabular}

$B N P$ B-type natriuretic peptide, $R A S i$ renin-angiotensin system inhibitor, $C C B$ calcium channel blocker, $L V E F$ left ventricular ejection fraction, $C A D$ coronary artery disease

in this study, and DBP measures could have changed after measurement. Third, we diagnosed HFpEF according to the echocardiographic data obtained within $24 \mathrm{~h}$ after admission. We did not have data at the compensated phase of HF, which may have affected the patient cohort.

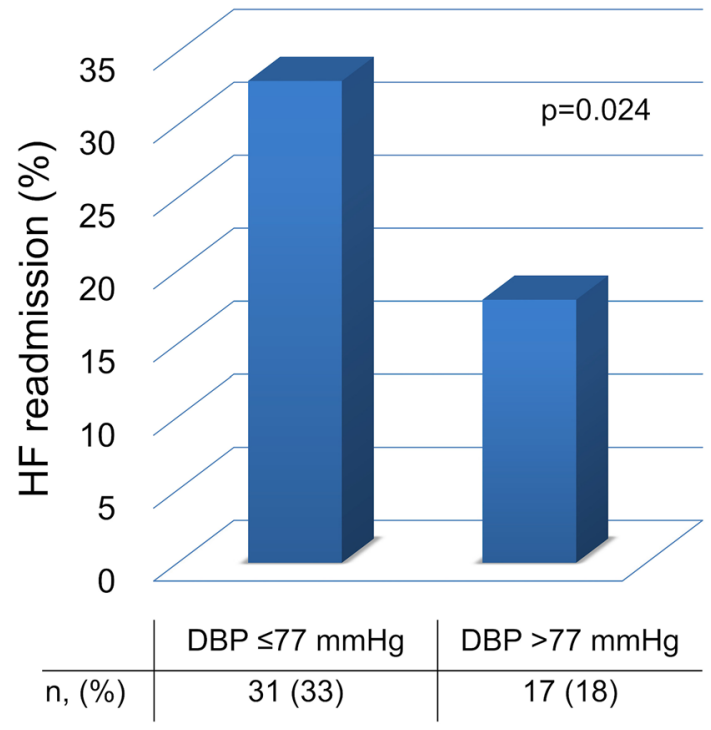

Fig. 2 Incidence of heart failure readmission according to diastolic blood pressure level-low diastolic blood pressure was related to an increased risk of heart failure readmission in patients with heart failure with preserved ejection fraction

\section{Conclusion}

Low DBP was independently associated with HF readmission in hospitalized patients with decompensated HFpEF. Our findings suggest that DBP may be a useful risk-stratification tool in this population.
Fig. 3 Kaplan-Meier analysis of diastolic blood pressure in patients with heart failure with preserved ejection fractionlow diastolic blood pressure (DBP $\leq 77 \mathrm{mmHg}$ ) predicted $\mathrm{HF}$ readmission (green line). Blue line, $\mathrm{DBP}>77 \mathrm{mmHg}$

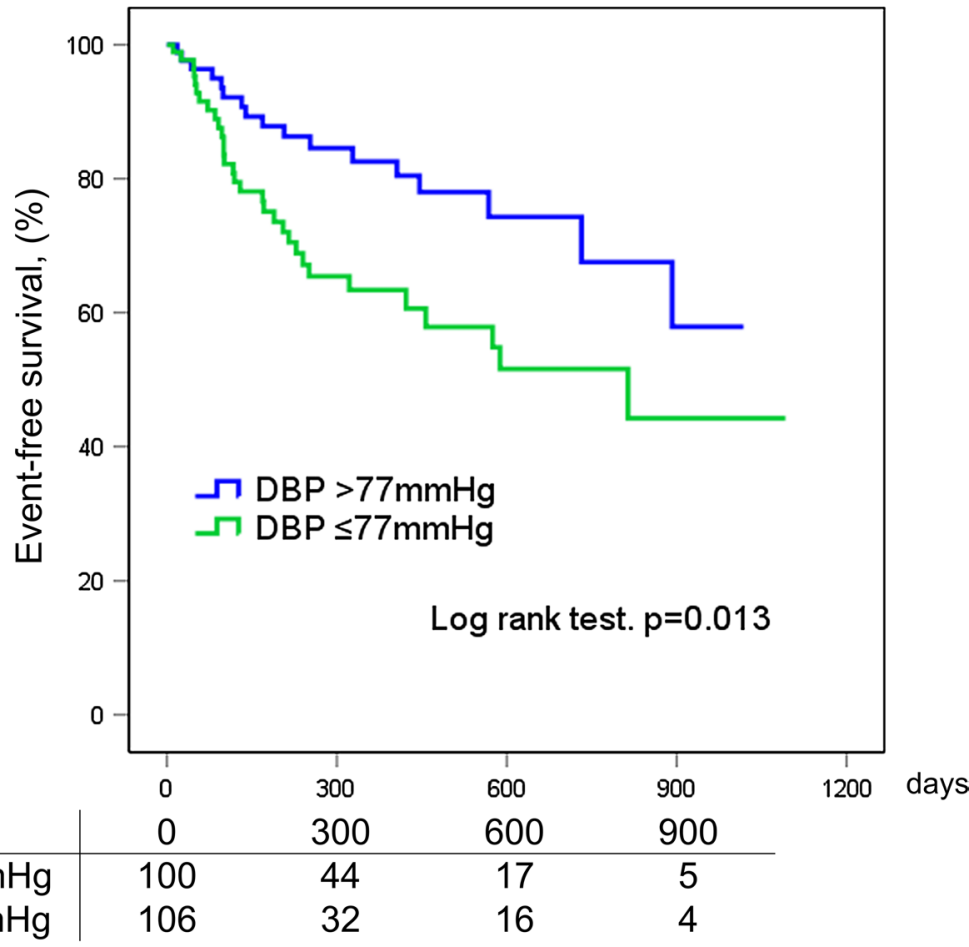


Table 3 Multivariable Cox proportional hazard analysis

\begin{tabular}{lll}
\hline Variables & Hazard ratio (95\% CI) & $p$-value \\
\hline $\begin{array}{l}\text { Diastolic blood pres- } \\
\text { sure } \leq 77 \mathrm{mmHg}\end{array}$ & $2.229(1.021-4.867)$ & 0.044 \\
Age (years) & $1.019(0.973-1.068)$ & 0.420 \\
Male, sex & $1.459(0.702-3.039)$ & 0.310 \\
Systolic blood pressure (mmHg) & $0.987(0.973-1.001)$ & 0.072 \\
Hemoglobin (g/dL) & $1.021(0.889-1.173)$ & 0.765 \\
Serum albumin (g/dL) & $0.807(0.400-1.627)$ & 0.548 \\
Serum creatinine (mg/dL) & $1.092(0.629-1.898)$ & 0.754 \\
BNP (pg/mL) & $1.000(0.999-1.001)$ & 0.527 \\
RASis, $n(\%)$ & $0.996(0.503-1.969)$ & 0.990 \\
CCBs, $n(\%)$ & $0.744(0.360-1.538)$ & 0.425 \\
LVEF $(\%)$ & $0.981(0.928-1.036)$ & 0.490 \\
CAD, $n(\%)$ & $1.006(0.465-2.178)$ & 0.988 \\
Living alone, $n(\%)$ & $1.213(0.359-1.865)$ & 0.646 \\
\hline
\end{tabular}

$B N P$ B-type natriuretic peptide, RASi renin-angiotensin system inhibitor, $C C B$ calcium channel blocker, $L V E F$ left ventricular ejection fraction, $C A D$ coronary artery disease, $C I$ confidence interval

\section{Acknowledgements None.}

\section{Compliance with ethical standards}

\section{Conflict of interest None.}

Open Access This article is licensed under a Creative Commons Attribution 4.0 International License, which permits use, sharing, adaptation, distribution and reproduction in any medium or format, as long as you give appropriate credit to the original author(s) and the source, provide a link to the Creative Commons licence, and indicate if changes were made. The images or other third party material in this article are included in the article's Creative Commons licence, unless indicated otherwise in a credit line to the material. If material is not included in the article's Creative Commons licence and your intended use is not permitted by statutory regulation or exceeds the permitted use, you will need to obtain permission directly from the copyright holder. To view a copy of this licence, visit http://creativecommons.org/licenses/by/4.0/.

\section{References}

1. Okura Y, Ramadan MM, Ohno Y, Mitsuma W, Tanaka K, Ito M, Suzuki K, Tanabe N, Kodama M, Aizawa Y (2008) Impending epidemic: future projection of heart failure in Japan to the year 2055. Circ J 72:489-491

2. Owan TE, Hodge DO, Herges RM, Jacobsen SJ, Roger VL, Redfield MM (2006) Trends in prevalence and outcome of heart failure with preserved ejection fraction. N Engl J Med 355:251-259

3. Yasuda S, Miyamoto Y, Ogawa H (2018) Current status of cardiovascular medicine in the aging society of Japan. Circulation 138:965-967

4. Shimokawa H, Miura M, Nochioka K, Sakta Y (2015) Heart failure as a general pandemic in Asia. Eur J Heart Fail 17:884-892

5. Witteman JC, Grobbee DE, Valkenburg HA, van Hemert AM, Stijnen T, Burger H, Hofman A (1994) J-shaped relation between change in diastolic blood pressure and progression of aortic atherosclerosis. Lancet 343:504-507

6. Shiomura R, Kobayashi N, Shirakabe A, Okazaki H, Shibata Y, Yasushi M, Noritake H, Asai K, Shimizu W (2020) Systolic blood pressure and cardiac mortality related to serum total bilirubin levels at admission in patients with acute heart failure. Heart Vessels. https://doi.org/10.1007/s00380-020-01666-1

7. Sandesara PB, O'Neal WT, Kelli HM, Topel M, Samman-Tahhan A, Sperling LS (2018) Diastolic blood pressure and adverse outcomes in the TOPCAT (Treatment of preserved cardiac function heart failure with an aldosterone antagonist) trial. J Am Heart Assoc 7:e007475

8. Tsujimoto T, Kajio H (2018) Low diastolic blood pressure and adverse outcomes in heart failure with preserved ejection fraction. Int J Cardiol 263:69-74

9. McKee PA, Castelli WP, McNamara PM, Kannel WB (1971) The natural history of congestive heart failure: the Framingham study. N Engl J Med 285:1441-1446

10. Lang RM, Badano LP, Mor-Avi V, Afilalo J, Armstrong A, Ernande L, Flachskampf FA, Elyse Foster E, Goldstein SA, Kuznetsova T, Lancellotti P, Muraru D, Picard MH, Rietzschel ER, Rudski L, Spencer KT, Tsang W, Voigt JU (2015) Recommendations for cardiac chamber quantification by echocardiography in adults: an update from the American Society of Echocardiography and the European Association of Cardiovascular Imaging. Eur Heart J Cardiovasc Imaging 16:233-270

11. Nagueh SF, Smiseth OA, Appleton CP, Byrd BF 3rd, Dokainish H, Edvardsen T, Flachskampf FA, Gillebert TC, Klein AL, Lancellotti P, Marino P, Oh JK, Popescu BA, Waggoner AD, AD, (2016) Recommendations for the evaluation of left ventricular diastolic function by echocardiography: an update from the American Society of Echocardiography and the European Association of Cardiovascular Imaging. J Am Soc Echocardiogr 29:277-314

12. Banach M, Bhatia V, Feller MA, Mujib M, Desai RV, Ahmed MI, Guichard JL, Aban I, Love TE, Aronow WS, White M, Deedwania P, Fonarow G, Ahmed A (2011) Relation of baseline systolic blood pressure and long-term outcomes in ambulatory patients with chronic mild to moderate heart failure. Am J Cardiol 107:1208-1214

13. Rich JD, Burns J, Freed BH, Maurer MS, Burkhoff D, Shah SJ (2018) Meta-Analysis Global Group in Chronic (MAGGIC) heart failure risk score: validation of a simple tool for the prediction of morbidity and mortality in heart failure with preserved ejection fraction. J Am Heart Assoc. https://doi.org/10.1161/JAHA.118.009594

14. Balmain S, Padmanabhan N, Ferrell WR, Morton JJ, McMurray JJ (2007) Differences in arterial compliance, microvascular function and venous capacitance between patients with heart failure and either preserved or reduced left ventricular systolic function. Eur J Heart Fail 9:865-871

15. Karagodin I, Aba-Omer O, Sparapani R, Strande JL (2017) Aortic stiffening precedes onset of heart failure with preserved ejection fraction in patients with asymptomatic diastolic dysfunction. BMC Cardiovasc Disord 17:62

16. Aizawa Y, Okumura Y, Saito Y, Ikeya Y, Nakai T, Arima K (2020) Association of renal resistance index and arterial stiffness on clinical outcomes in patients with mild-to-moderate renal dysfunction and presence or absence of heart failure with preserved ejection fraction. Heart Vessels 35:1699-1708

17. Bots ML, Witteman JC, Hofman A, de Jong PT, Grobbee DE (1996) Low diastolic blood pressure and atherosclerosis in elderly subjects: the Rotterdam study. Arch Intern Med 156:843-848

18. Franklin SS, Gustin W 4th, Wong ND, Larson MG, Weber MA, Kannel WB, Levy D (1997) Hemodynamic patterns of age-related changes in blood pressure. Circulation 96:308-315

19. Hashimoto J, Ito S (2017) Central diastolic pressure decay mediates the relationship between aortic stiffness and myocardial 
viability: potential implications for aortosclerosis-induced myocardial ischemia. J Hypertens 35:2034-2043

20. McEvoy JW, Chen Y, Rawlings A, Hoogeveen RC, Ballantyne CM, Blumenthal RS, Coresh J, Selvin E (2016) Diastolic blood pressure, subclinical myocardial damage, and cardiac events: implications for blood pressure control. J Am Coll Cardiol 68:1713-1722

21. Chirinos JA, Kips JG, Jacobs DR Jr, Brumback L, Duprez DA, Kronmal R, Bluemke DA, Townsend RR, Vermeersch S, Segers P (2012) Arterial wave reflections and incident cardiovascular events and heart failure: MESA (Multiethnic Study of Atherosclerosis). J Am Coll Cardiol 60:2170-2177

22. Stamler J, Stamler R, Neaton JD (1993) Blood pressure, systolic and diastolic, and cardiovascular risks. US population data Arch Intern Med 153:598-615
23. Flint AC, Conell C, Ren X, Banki NM, Chan SL, Rao VA, Melles RB, Bhatt DL (2019) Effect of systolic and diastolic blood pressure on cardiovascular outcomes. N Engl J Med 381:243-251

Publisher's Note Springer Nature remains neutral with regard to jurisdictional claims in published maps and institutional affiliations. 